\title{
Generating Schematic Cable Plans Using Springembedder Methods
}

\author{
Ulrich Lauther and Andreas Stübinger \\ Siemens AG, CT SE 6, Munich \\ \{Ulrich.Lauther, Andreas. Stuebinger\}@mchp.siemens.de
}

Motivation and Area of Application. For documentation of communication networks like telephon networks normaly there are two different representations: a ground plan showing the exact coordinates of all net elements (man holes, trenches, cables cabinets, exchanges, ... ), usually stored and managed in a GIS system, and a schematic plan containing the same information, but drawn in a schematic way, not properly scaled, but in an easier to understand way. Another kind of schematic maps are often used to display connections in a urban public transportation system: the arrangement of train stations shows some similiarity to geographic coordinates, but the main importance is to show an easy to understand drawing of the connection possibilities by using a more orthogonal drawing.

In telecomunication GIS systems it is possible to handle both views of the net: the ground plan, and a schematic plan. However, this implies a high burdon on keeping both plans consistent up to date. Every time a connection is inserted, updated or deleted in the ground plan it has to be re-done in the schematic map, too. Therfore, updateing both plans is a very tedious task and very prone to errors.

This burdon is resolved by an automatic schematic map generation algorithm described in this paper. Instead of updating two different views in lockstep, only one map, the ground plan, is updated manually. The schematic map then is generated automaticly by SCHEMAP.

Input data. The input for SCHEMAP consists of the ground plan, containing two different but highly interwoven graphs: a track graph, describing man holes and duct elements, and a cable graph describing the electrical components of the telecomunication network. The cable graph is embedded inside the track graph as all cables have to lie inside some trenches. In the case of an air cable a dummy duct is created in the track graph.

Layout Algorithms and Constraints to honor. The track layout is done by a modified springembedder based on [1, the cable layout by a specialized sorting algorithm and the track node layout (i.e. cable nodes inside a track node) by a combination of pattern router and Lee router.

To get a schematic view of the input graph the algorithm has to take the following constraints into account:

P. Mutzel, M. Jünger, and S. Leipert (Eds.): GD 2001, LNCS 2265, pp. 465-466, 2002.

(C) Springer-Verlag Berlin Heidelberg 2002 
Similarity of location. Crossings in the input have to be preserved; however, no new crossings may be added. Also, north/south and east/west relations have to be preserved.

Orthogonal. An "as orthogonal as possible" drawing should be achieved.

Sizes for nodes and edges. To draw cable edges inside the track edges a track edge has to have some size. This implies that track nodes get a rectangle box having some size to connect a track edge.

Results. In figure 1 on the left a typical input for SCHEMAP is shown. Only the track graph is visible, all the cables are hidden, as there are no coordinates given for the cable graph elements. The boxes near the track edges represent textlabels. On the right of figure 1 the output of SCHEMAP is shown. Nearly all track edges are orthogonal, all cable edges are sorted.
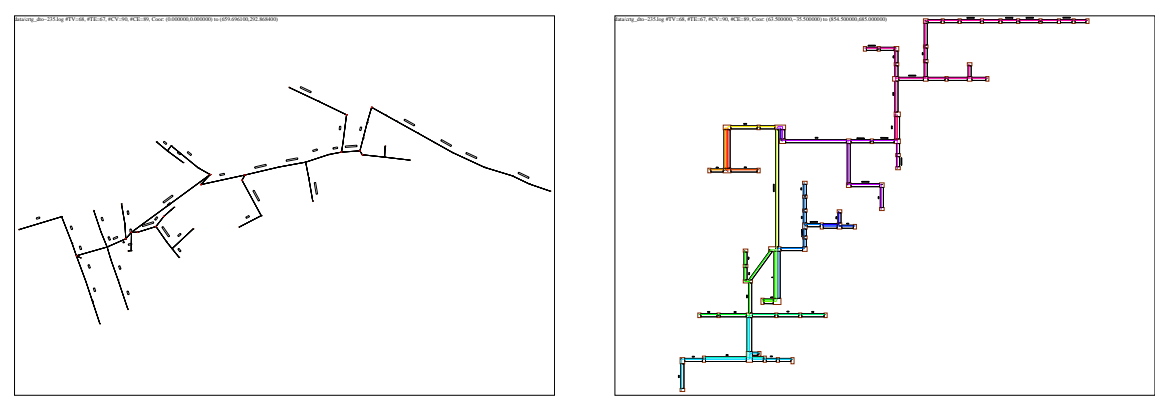

Fig. 1. Left: Typical input ground plan, Right: Resulting schematic view. To show the cables in more detail different colors are chosen.

The drawings are normaly not perfect in every sense, i.e. normaly there are some minor modifications to be done by hand. However, SCHEMAP achieves its goal pretty good: the schematic drawings show the input graph in a more schematic way respecting all the given constraints and it is fast enough to be used in daily work. SCHEMAP is implemented in $\mathrm{C}++$ using the TURBO library (see [3]) and is integrated as module SCHEMAP into NetMinisterOSP (see [2]).

\section{References}

1. T.M.J. Fruchterman, E.M. Reingold: Graph Drawing by Force-Directed Placement, Software - Practice and Experience, Vol. 21, pp. 1129 - 1164, 1991.

2. NetMinister-OSP, Order No. A50001-N12-P14-1-7600, Siemens AG, 2000.

3. U. Lauther: TURBO - In Projekten schneller zum Ziel, Software@Siemens, 1999. 